Fecha de recepción: noviembre 2019 Fecha de aceptación: diciembre 2019 Versión final: marzo 2020

\section{Propuesta curricular como estrategia de innovación educativa: carrera de Diseño de Productos de la PUCE Ecuador}

Caridad González Maldonado ${ }^{(1)}$

\begin{abstract}
Resumen: En el siguiente artículo se describen algunos de los motivos principales que impulsan el cambio curricular en la formación superior universitaria de la Pontificia Universidad Católica del Ecuador. Ello a la luz principalmente de circunstancias nacionales socioeconómicas y reformas educativas de tipo legislativas. Se indaga entonces en la propuesta del rediseño curricular que se establece en el año 2019 para la carrera de Diseño de Productos de la institución en cuestión y con un enfoque que pone la mirada en las estrategias al interior de la misma en el marco de la innovación educativa. Se abordan factores como los ambientes virtuales, las Tecnologías de la Información y la Comunicación, la disminución de los períodos académicos de la mano de una selección de contenidos sustanciales, entre otros. Finalmente se concluye que los factores de innovación desarrollados parecen organizarse por capas definidas desde instancias nacionales, institucionales (PUCE) y al interior de la propia carrera, a razón de lo cual se debe reflexionar sobre el modo en que esta última se puede apropiar del discurso y proceso de cambio para articular sus propias acciones innovadoras y significativas.
\end{abstract}

Palabras clave: currículo académico - Diseño de Productos - Educación Superior - formación universitaria - innovación curricular.

[Resúmenes en inglés y portugués en las páginas 162-163]

(1) Adscrita a la Pontificia Universidad Católica del Ecuador, Quito, en la Carrera de Diseño de Productos de la Facultad de Arquitectura, Diseño y Artes. Diseñadora Industrial con Máster en Gestión e Innovación de Diseño y candidata a Doctor en Diseño por la Universidad de Palermo, Buenos Aires. Coautora del libro "Pertinencia Curricular en la Educación Superior Tecnológica”. Artículos: Discurso visual en la comunicación gubernamental del Ecuador: propuesta de análisis; Diseño y política en Ecuador hipermediático: Marca País y comunicación gubernamental. Experiencia docente en el Instituto Superior de Diseño, Universidad de La Habana, Cuba y en Ecuador en instituciones como: Universidad Internacional del Ecuador, Universidad de las Américas, Universidad UTE. Actualmente Docente e Investigadora en la PUCE. 


\section{Introducción}

La Educación Superior en Ecuador se vincula con el proceso constante y formativo que tiene lugar a lo largo de la vida. Su "carácter humanista, intercultural y científico constituye un derecho de las personas y un bien social que... responderá al interés público y no... al servicio de intereses individuales y corporativos" (LOES, 2 de agosto de 2018, p. 7). En tal virtud la formación educativa se centra en el ser humano, en pro de garantizar su desarrollo holístico (Constitución de la República del Ecuador, 2008) pero también comprometido con los objetivos nacionales y los Planes de Desarrollo del Estado es decir, con la visión política, por lo que es relevante tomar en cuenta toda la complejidad contextual donde se desenvuelve y que le atraviesa (Fägerlind y Saha, 1983; Larrea de Granados, 2015).

Esto supone un reto para la integración entre la Universidad, el Estado y la sociedad, a la vez que marca las pautas para la generación de un modelo de currículo que independientemente de sus etapas de configuración (ver figura 1) se entiende como un "sistema complejo, abierto y vivo" (Herrera et al, 2000, p. 2) que orienta y organiza el proceso de enseñanza-aprendizaje.

\section{Estructuras para el desarrollo curricular}

\begin{tabular}{lll}
\hline $\begin{array}{l}\text { Arnaz } \\
\mathbf{( 1 8 9 1 )}\end{array}$ & $\begin{array}{l}\text { Diaz } \\
(\mathbf{1 9 9 0 )}\end{array}$ & $\begin{array}{l}\text { Vargas (2008); } \\
\text { TEC(2015) }\end{array}$ \\
\hline Elaborar & Fundamentación & Diseño \\
\hline Instrumentar & Delimitación del perfil de egreso & Desarrollo \\
\hline Aplicar & Organización y estructuración & Gestión \\
\hline Evaluar & Evaluación continua & \\
\hline
\end{tabular}

Figura 1. Denominaciones comparadas sobre las etapas para el desarrollo curricular. Elaboración propia. Nota. (TEC) se refiere al Tecnológico de Monterrey.

Es en este proceso de enseñanza-aprendizaje dentro del campo formativo que se contextualiza el término de innovación educativa, el cual según la UNESCO (2014) se refiere a:

un acto deliberado y planificado de solución de problemas, que apunta a lograr mayor calidad en los aprendizajes de los estudiantes, superando el paradigma tradicional. Implica trascender el conocimiento academicista y pasar del aprendizaje pasivo del estudiante a una concepción donde el aprendizaje es interacción y se construye entre todos. (p.3) 
El académico español Pedro Cañal de León en su obra "La Innovación educativa” (2002) menciona que esta es un proceso y no una actividad puntual que incluye un "conjunto de ideas... y estrategias, más o menos sistematizadas, mediante las cuales se trata de introducir y provocar cambios en las prácticas educativas vigentes" (p.11).

Los autores Margalef y Arenas (2006) retomando a Zaltman y otros (1973) agregan que "el concepto de innovación aparecerá relacionado a tres usos: la creación de algo desconocido, la percepción [vinculación del pensamiento y la conducta] de lo creado como algo nuevo y la asimilación de ese algo como novedoso [práctica, uso, decisión]" (p.1).

En acuerdo con los autores antes citados, innovar viene de la mano de acciones y por tanto de stakeholders ${ }^{1}$ (actores implicados) en un proceso compartido de intervención y cambio que debe alinearse a la realidad en base al compromiso socio-educativo con la formación del ser humano. Esto a nivel de currículo se sintetiza en el perfil de egreso, documento para la construcción del plan de estudios que sobre la base de las demandas productivas, económicas, empresariales, estatales, públicas, entre otras permite en conjunto definir las competencias, destrezas, valores y actitudes esperadas en el profesional.

En efecto, el currículo constituye una de las bases fundamentales de la concepción integrada de la educación "que carga con una multiplicidad de agendas ...que se superponen y muchas veces entran en colisión entre sí...reflejo de proyectos e intereses distintos” (p.4). En este punto es válido agregar en acuerdo con José Antonio Arnaz (1981) que:

Todo currículum es necesariamente una abstracción, pues en su elaboración no es posible, ni deseable considerar todos los aspectos, todas las variables, la totalidad de alternativas; se toma en consideración solo lo que se juzga (bien o mal) como lo más importante. (p. 35)

En medio de estos desafíos el presente estudio se interesa en el modo en que la carrera de Diseño de Productos ${ }^{2}$ de la Pontificia Universidad Católica del Ecuador pretende dar respuestas a la innovación desde su propuesta de rediseño curricular. Es importante entonces conocer los enfoques, modelo y principios educativos que rigen en la institución a nivel macrocurricular, para desde la directriz de los mismos enfocar lo meso y microcurricular. A partir de la lógica del currículo situado en contexto, la Pontificia Universidad Católica del Ecuador (PUCE) se ratifica en su intencionalidad educativa de aprendizaje Basado en Competencias. Este enfoque, según se indica en la red iberoamericana de colaboración universitaria Universia: "erradica el modelo tradicional que basa el aprendizaje en la memorización de datos e información, que muchas veces resulta irrelevantes para la vida real" (Universia, 30 de noviembre de 2018) y establece que:

Una competencia es un conocimiento en ejecución y funciona como una habilidad para hacer frente a determinadas situaciones en cualquier ámbito de la vida. El modelo educativo basado en competencias atiende al proceso educativo del estudiante, más que su complimiento con el curso, lo que permite el desarrollo integral en cada joven. En el proceso, se genera un escenario parti- 
cipativo en el que los estudiantes dejan de ser meros receptores de información para pasar a ser agentes activos. Son responsables de su propio aprendizaje, lo que genera una fuerte autonomía. (Párrs.3-5)

Por tanto, la Educación Basada en Competencias (EBC) se orienta a una educación que busca de manera efectiva enfrentar al estudiante con las diversas realidades del contexto poniendo atención en un saber hacer que favorezca el logro de sus resultados. Sin embargo, la mejora del rendimiento en alineación con la economía nacional y con los retos de la competitividad se plantea también de manera crítica (Barrón, 2000; Rojas, 2000; Barnett, 2001; Guzmán, 2003) bajo el postulado de que el EBC implica una "visión productivista (propia) de la globalización neoliberal" (Echavarría y De Los Reyes, 2017, p. 2), donde "el ideal del hombre es el...sometido a procesos de capacitación y reciclaje continuo" (Barón, 2000, p. 6) que favorecen la perspectiva economicista-empresarial por sobre la reflexión intelectual.

A razón de lo anterior es importante describir el (EBC) a partir de sus escenarios principales, de manera que se clarifique de los mismos el enfoque adoptado por la Pontificia Universidad Católica del Ecuador y que puntualmente interesa para el presente estudio, sobretodo porque el camino asumido por la institución se concreta en el diseño curricular de su oferta académica.

Según afirman los investigadores cubanos María J. Vidal, Ramón Salas, Bertha Fernández y Ana Liz García (2016) el fundamento de las competencias se formula:

Desde el escenario laboral...y desde el mundo de la educación. En el mundo del trabajo, la competencia es una capacidad que solo puede desplegarse en una situación concreta del desempeño laboral, el cual tiene sus reglas, escenarios, procedimientos, instrumentos y consecuencias; mientras que en las universidades aunque responde a una misma idea básica, su demostración se despliega solamente en situaciones de evaluación educacional, donde se va verificando cómo avanzan los educandos en su aprendizaje a través de la construcción de los modos de actuación profesional. (p.5)

Por su parte Laura Echavarría Canto (2010) sobre la genealogía de la Educación Basada en Competencias refiere que:

...existen tres modelos: el británico (años ochenta) cuyo énfasis radica en los aspectos evaluativos de las competencias que se definen en función de las normas necesarias en el medio laboral conocidas como NVQ (National Competente Vocational Qualification $)^{3}$ que tiene cinco niveles; el Australiano, cuyo énfasis está en la interrelación de los atributos lo que da un gran peso al juicio profesional; y el Español (1993) que gira en torno al saber hacer y a los procedimientos. (p.150) 
En relación a los enfoques planteados para el EBC no se puede afirmar que la PUCE en Ecuador asuma una postura puntual (aunque parece más propia la referencia a la tipología española), ya que como se puede ver la implementación del modelo es flexible según las particularidades e intereses de cada institución laboral o educativa, lo que conlleva a una adaptación del modelo a sus propios principios.

En este sentido los principios de la PUCE en los cuales se ampara su propuesta de (EBC) y que le dan identidad propia son: a) la centralidad del estudiante, que fomenta el desarrollo de las cualidades necesarias para que este enfrente eficazmente su proceso formativo subrayando la primacía del aprendizaje sobre la enseñanza, b) el humanismo cristiano, que propone a Jesucristo como modelo de vida sin intención impositiva o discriminatoria promoviendo valores para el crecimiento personal, el servicio a los demás y a favor de la reconciliación con la naturaleza como ser vivo digno de respeto c) la pedagogía ignaciana, que orienta el proceso educativo a través de contextualizar la realidad, experimentarla vivencialmente, reflexionar sobre esa experiencia, actuar consecuentemente y evaluar la acción y el proceso seguidos; y d) la integración del saber, que reconoce y valora los saberes no formales y la práctica de la interculturalidad dentro de la universalidad de conocimientos producidos a lo largo del tiempo sin dejar de lado su enfoque afín a los campos de actuación del profesional (Modelo Educativo de la PUCE, 6 de marzo de 2017).

Por lo anterior la universidad entiende las consecuencias que implica tener un currículo desligado de la realidad y se emprende en un proceso de valoración y cambio curricular desde el año 2017 que involucra sus 13 Facultades. Este cambio se incorpora paulatinamente a la luz de las demandas modernas y los requerimientos socio-políticos y económicos que dentro de un marco de pertinencia nacional favorecen a futuro la sostenibilidad de las carreras y su calidad dentro de la institución educativa. En lo que se verá a continuación se hace referencia a la situación nacional.

\section{Antecedentes: realidad nacional de cara a la coyuntura laboral y a la nueva legislación educativa}

La Educación Superior en Ecuador ha transitado por diversas transformaciones desde la última década. El 6 de junio del año 2019 el Presidente Constitucional Lenín Moreno Garcés expide de manera oficial en el país el Reglamento General a la Ley Orgánica de Educación Superior (LOES), el cual establece ajustes sobre la versión que le precede del 12 de octubre de 2010 concebida bajo el Gobierno del expresidente Rafael Correa.

Uno de los aspectos más relevantes que se establece en la legislación del 2019 es el vinculado a la creación en el país de "carreras...especializadas en la formación técnica y tecnológica [habilitadas] para emitir títulos de tercer nivel superior y de postgrado tecnológico" (Reglamento General a la LOES, 2019, p. 9). En apoyo a esta medida también se plantea que los programas educativos creados "accedan a procesos simplificados de aprobación [por el Consejo de Educación Superior ${ }^{4}$ ]...en un plazo no mayor a 30 días" (Reglamento General a la LOES, 2019, p. 10). 
"Estos cambios forman parte del plan nacional de fortalecimiento y revalorización de la formación técnica y tecnológica” (Metro Ecuador, 27 de febrero de 2019) en el país, lo que representa entre otros elementos una disminución de las brechas educativas y una proyección de mejora en las oportunidades laborales y en la remuneración para los profesionales vinculados a este tipo de formación que a la fecha se concreta en 3 años de estudios.

La medida se traduce según la Secretaría de Educación Superior, Ciencia, Tecnología e Innovación del Ecuador (SENESCYT) en una oferta educativa de instituciones de tercer nivel que para el segundo semestre del año 2019 incrementa tres veces más (316\%) la cifra histórica enmarcada particularmente en Universidades y Escuelas politécnicas (ver figura 2).

\begin{tabular}{llcl}
\multicolumn{4}{c}{ Oferta de Instituciones de Educación Superior en Ecuador } \\
30 & $\begin{array}{l}\text { Universidades y escuelas } \\
\text { politécnicas públicas }\end{array}$ & 85 & $\begin{array}{l}\text { Institutos superiores técnicos, } \\
\text { tecnológicos y conservatorios } \\
\text { públicos }\end{array}$ \\
\hline 26 & $\begin{array}{l}\text { Universidades } \\
\text { particulares }\end{array}$ & 92 & $\begin{array}{l}\text { Institutos superiores técnicos, } \\
\text { tecnológicos y conservatorios } \\
\text { particulares }\end{array}$ \\
\hline 56 & Total & 177 & Total \\
\hline
\end{tabular}

Figura 2. Clasificación del total de instituciones con oferta de tercer nivel en Ecuador. Elaboración propia. Fuente (SENESCYT, 2019). Recuperado de http://admision.senescyt.gob.ec/

Igualmente en el suplemento informativo en línea "Oferta académica de las instituciones de Educación Superior" (SENESCYT, 2019) que orienta a los potenciales estudiantes de tercer nivel de manera abierta y oficial sobre los procesos para la postulación a carreras a nivel nacional, se encuentran propuestas gráficas a modo de bandas horizontales en la zona inferior de algunas de sus páginas, que promueven particularmente el ingreso estudiantil a centros técnicos y tecnológicos, no así para centros universitarios o politécnicos (ver figura 3). 


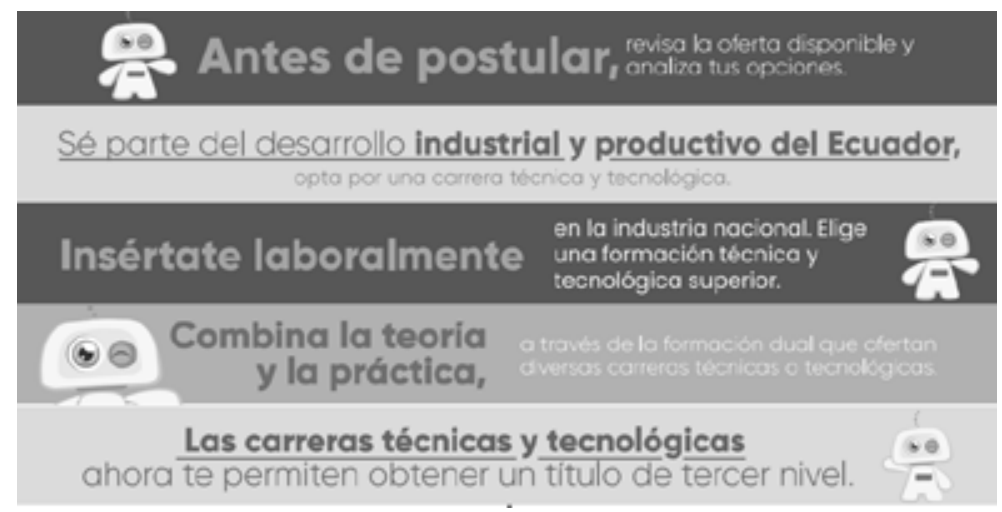

Figura 3. Bandas gráficas de la 1 al 5 para promover el ingreso a carreras técnicas y tecnológicas. Elaboración propia. Fuente (SENESCYT, 2019). Recuperado de http://admision.senescyt.gob.ec/

Esto muestra acciones comunicativas por el Gobierno Nacional consecuentes con las medidas establecidas. A la vez demanda que las universidades incorporen procesos de mejora continua que evidencien, que dentro del cambio propuesto, el rol del profesional universitario, en tanto generador de propuestas debe articularse a la ejecución de técnicos y tecnólogos, en una lógica equilibrada y cohesionada dirigida a potenciar el desarrollo nacional. Sin embargo, como parte de este proceso las instituciones universitarias y politécnicas mayormente con formación de 5 años, se ven afectadas en términos de demanda de carreras y tasa de retención estudiantil, lo que se manifiesta en una deserción temporal o completa de los educandos vinculada principalmente a las variables de tiempo de egreso, con 2 años adicionales a los de la formación técnica y costos económicos de mayor cuantía para estudios de pregrado en nivel universitario donde el ingreso se encuentra "...fuertemente condicionado por el origen socioeconómico de las y los estudiantes” (CINDA 2011, p.168).

Se suma a esta circunstancia la supresión de la "categorización de las universidades y se da paso a la acreditación" (El comercio, 9 de julio de 2019, párr. 4). En el caso de la categorización universitaria esta se pautó en la Ley del año 2010 en tanto acción vinculada al principio de calidad y en la búsqueda constante por la excelencia.

Según se registra en la LOES (12 de octubre de 2010):

La Acreditación es una validación de vigencia quinquenal realizada por el Consejo de Evaluación, Acreditación y Aseguramiento de la Calidad de la Educación Superior [actualmente CASES], para certificar la calidad de las instituciones de educación superior, de una carrera o programa educativo, sobre la base de una evaluación previa.

...es el producto de una evaluación rigurosa sobre el cumplimiento de lineamientos, estándares y criterios de calidad de nivel internacional, a las carreras, programas, postgrados e instituciones, obligatoria e independiente... incluye 
una autoevaluación de la propia institución, así como una evaluación externa realizada por un equipo de pares expertos, quienes a su vez deben ser acreditados periódicamente. (Art. 95, p. 37)

La clasificación académica o categorización de las instituciones, carreras y programas será el resultado de la evaluación. Hará referencia a un ordenamiento de las instituciones, carreras y programas de acuerdo a una metodología que incluya criterios y objetivos medibles y reproducibles de carácter internacional. (Art. 97, p. 37)

En el Diario nacional El telégrafo (27 de noviembre de 2013) sobre el sistema de categorización se menciona además que:

El modelo de evaluación que se utilizó para la categorización se basa en los criterios de: academia, eficiencia académica, investigación, organización e infraestructura. En total fueron 46 indicadores los que se utilizaron para hacer este informe. Las categorías ${ }^{5}$ son las siguientes: A. con un desempeño superior al promedio del sistema. B: se encuentra muy cerca del promedio y por encima de él. C: se encuentra cerca del promedio, pero debajo de él. D: presentan valores de desempeño por debajo del promedio. Las instituciones educativas que están en las categorías A, B o C están acreditadas dentro del Sistema de Educación Superior, mientras que las que se ubican en la D tienen que cumplir con un plan de fortalecimiento institucional que les permita ubicarse por lo menos en categoría $\mathrm{C}$, en un plazo máximo de dos años. (Párr.3-5)

Revertir el proceso de categorización (A, B, C o D) tiene como finalidad efectuar procesos más dinámicos dirigidos a evaluar las condiciones básicas esenciales de las Instituciones de Educación Superior (IES) en Ecuador. Además propicia que las mismas se autoevalúen desde modelos ajustados a sus propias realidades y particularidades (Ecuador Universitario, 5 de septiembre de 2019).

Sin negar lo planteado y aunque se registren asimetrías entre universidades por ejemplo según niveles de modernización tecnológica, infraestructura respecto a otras en procesos de búsqueda y transición hacia esos estándares; se considera que la supresión de categorías universitarias eliminó la motivación de cumplir de manera eficiente con procesos internos competitivos que buscaban concretar mejoras continuas a nivel institucional con miras a sobrepasar las denominadas por el Gobierno "condiciones básicas de calidad" de aparente enfoque general y superficial.

Adicionalmente el valor simbólico que se adquiría con el otorgamiento de las calificaciones generaba un incentivo en el esfuerzo familiar por matricular a los jóvenes en instituciones de prestigio, con presencia internacional, lo cual significaría mejores posibilidades de financiamiento para realizar estudios complementarios a la formación, cursos abiertos, cursos de verano con doble titulación, postulación a becas de postgrado, además de mejores oportunidades para aplicar a viajes de intercambio estudiantil, vínculo con redes académicas internacionales lo que como consecuencia podría representar una fortaleza en la hoja de vida al acceder al mercado laboral. 
En otro orden de ideas y en relación al empleo e ingreso económico en el país, es importante puntualizar que en Ecuador los sectores reconocidos por el sistema económico que opera bajo el modelo capitalista son: el sector público, el privado y el de la economía popular y solidaria. La articulación de estos tres sectores es la que mueve el desarrollo de la economía nacional vinculada a procesos de producción, financiamiento, distribución y consumo. "Desde esta perspectiva y respecto a la distribución del ingreso en el Ecuador, es notable la concentración de gran parte de la población en los niveles de ingresos bajos (el 49 por ciento percibe ingresos [mensuales] menores a USD 400.00)" (Alvarado y Pinos, 2017, p. 178). Este referente se confirma por el INEC (2019) el cual establece que "para septiembre 2019, el ingreso laboral promedio de un hombre con empleo es USD 369,1; mientras que para una mujer con empleo es de USD 312,9” (p. 48).

Si se parte del hecho de que "la educación superior pública puede cubrir solo el $51 \%$ de la demanda en Ecuador" (El Universo, 16 de junio de 2019), las cifras de ingreso laboral antes descritas reflejan un elevado contraste con el rango de costos por semestre que demandan las universidades privadas. Esto marca grandes diferencias de valor monetario que afectan la capacidad de pago para que los estudiantes accedan al sistema educativo universitario, lo que favorece la posibilidad de ingreso a institutos tecnológicos con costos de estudios más económicos.

Por ejemplo en el año 2014 el costo de algunas carreras en universidades privadas según el diario El Telégrafo (24 de mayo de 2014) se encontraba “entre \$2.000 y \$ 5.000 anuales más los cobros de matrícula” (Párr. 1). En el año 2019 y según indica la redactora del diario El Comercio Yadira Trujillo, el rango de costos semestral, independientemente de la posibilidad de acceso a una beca ${ }^{6}$, se definió entre $\$ 2.900$ y $\$ 5.300$ para carreras de ingeniería de las universidades San Francisco, Universidad UTE, Universidad de las Américas y PUCE. Esto igualmente no incluía pagos adicionales por inscripciones y matrículas (El Comercio, 24 de abril de 2019).

Por su parte la Secretaría General de la Pontificia Universidad Católica del Ecuador publicó de manera oficial en su página $\mathrm{Web}^{7}$ en el año 2018 la tabla de aranceles y matrículas por carreras. En la misma se indica que para el caso de estudios en Comunicación, Arte o Diseño los costos se encontraban entre \$2.125 semestrales con alguna beca asignada y $\$ 4.250$ sin goce de beca.

Estas circunstancias entre otras, marcaron pauta para ajustar el currículo de las carreras de la PUCE incluido el de Diseño de Productos, lo que se vio favorecido por la disposición transitoria ${ }^{8}$ oficial del Reglamento de Régimen Académico (RRA) en el país. Según lo establecido por el Consejo de Educación Superior CES y oficializado en la Gaceta ecuatoriana el 21 de marzo de 2019 el mismo establecía que:

A partir de la entrada en vigencia del presente Reglamento, y por una única vez, si las IES rediseñan sus carreras o programas vigentes, no vigentes y no vigentes habilitados para el registro de títulos sin que los ajustes impliquen cambios sustantivos, excepto a lo referente al criterio de duración, no será necesaria la aprobación por parte del CES. No obstante, las IES actualizarán los proyectos de carreras o programas y los remitirán al CES para su registro9. 
(RRA Disposición Transitoria Tercera, 2019)...Los proyectos serán presentados a través de la plataforma (en línea) del CES y contendrán la información y documentación establecida en la Guía metodológica para la presentación de carreras y programas ${ }^{10}$ que expida el CES. (Art. 121 RRA, 2019)... podrán acceder a este proceso las Universidades y Escuelas Politécnicas ubicadas en las categorías "A" y "B" y, los Institutos Superiores Acreditados. (RRA, 21 de marzo de 2019)

Sobre este reglamento cabe puntualizar que universidades como la PUCE, de categoría B, se acogen a la transitoria ya que la misma favorece la generación de cambios curriculares en tiempos mínimos y con procesos burocráticos simplificados. Esto en comparación con universidades de categorías $\mathrm{C}$ o $\mathrm{D}$ que aunque también podrán proponer sus cambios, dependen de procesos de aprobación más extensos y exigentes para poder implementar sus propuestas.

En el caso de Diseño de Productos como en la mayoría de las Facultades de la PUCE se definió como estrategia principal optimizar el tiempo de estudios, lo que a consecuencia implicaría una disminución de pagos arancelarios por parte de los estudiantes. Con esta perspectiva se planteó a nivel de acción reducir la malla curricular de carreras que venían históricamente ocurriendo en 10 o 9 semestres hacia carreras en 8 períodos académicos

\section{Proceso de cambio para la innovación: ajuste curricular en el Diseño de Productos de la PUCE}

En lo adelante se busca transparentar las acciones de innovación educativa tras el ajuste curricular como "palanca de innovación" (Castellanos, 2019) en la carrera de Diseño de Productos de la PUCE ubicada en Quito. Este se concreta en un plan de estudios que se codifica para su denominación como Q177.

La propuesta de innovación toma de base los parámetros que establece la Guía Metodológica para la Presentación de Carreras y Programas (CES, 24 de junio de 2019). Tales indicadores orientan y convergen en un modelo genérico de presentación de carreras que posteriormente se ajusta de acuerdo a los procesos y necesidades internas de cada institución. A partir de la Guía presentada y como parámetros de innovación, se describen en lo que sigue a continuación acciones establecidas al interior de la Pontificia Universidad del Ecuador, las cuales no se organizan por relevancia u orden de ejecución sino que responden a una secuencia que favorece la articulación de lo escrito.

En primer lugar la PUCE plantea adaptar su formación universitaria dentro de un rango de duración que permite estructurar el plan de estudios a partir de un mínimo de 5760 horas totales (para carreras en 8 períodos académicos) y un máximo de 7200 horas totales (para carreras en 10 períodos académicos). Ello en correspondencia con el rango de 40 a 60 cursos o asignaturas sugeridas respectivamente (ver figura 4) para integrar el proceso total de estudios. 


\begin{tabular}{|c|c|c|c|c|c|c|c|c|}
\hline \multirow[t]{2}{*}{$\begin{array}{l}\text { Tercer } \\
\text { nivel de } \\
\text { Grado }\end{array}$} & \multicolumn{2}{|c|}{$\begin{array}{l}\text { Duración en el } \\
\text { periodo } \\
\text { académico } \\
\text { ordinario (PAO) }\end{array}$} & \multicolumn{2}{|c|}{ Horas totales } & \multicolumn{2}{|c|}{ Créditos totales } & \multicolumn{2}{|c|}{$\begin{array}{l}\text { Número de } \\
\text { cursos o } \\
\text { asignaturas } \\
\text { sugerido } \\
\end{array}$} \\
\hline & Minimo & Máximo & Minimo & Máximo & Mínimo & Máximo & Mínimo & Máximo \\
\hline $\begin{array}{l}\text { Licenciatura } \\
\text { y titulos } \\
\text { profesionales }\end{array}$ & 8 & 10 & 5760 & 7200 & 120 & 150 & 40 & 60 \\
\hline
\end{tabular}

Figura 4. El PAO se refiere al período académico ordinario de 16 semanas de duración cada uno equivalente a 720 horas (RRA, art. 11, 2019).

El factor de innovación en este sentido se puede comprender a través de cómo se da particularmente respuesta a este condicionante de forma, sobretodo ya que se decide en específico por la carrera en cuestión asumir la opción de horas y períodos mínimos. Interesan entonces los criterios sobre los cuales se definen y priorizan los contenidos a impartir desde las asignaturas, así como aquellos que se fusiona o desechan teniendo en cuenta que "hay que ocuparse más por seleccionar contenidos sustanciales para el currículo y descubrir actividades académicas atractivas" (Sacristán y Pérez, 1997, p. 396) que les impulsen. En este punto la innovación educativa a nivel de carrera se volcó hacia la generación de grupos de trabajo por áreas o ejes formativos de interés: teórico, práctico, de representación y comunicación, materiales y procesos, que a través de procesos mediados por el consenso, incluso el disenso entre docentes internos, permitieron configurar la nueva propuesta de currículo.

En segundo lugar se orienta la formación a través de una estructura microcurricular dada por el CES, que establece y equilibra en horas la integración de los aprendizajes identificados según los siguientes componentes: aprendizaje en contacto con el docente, aprendizaje práctico experimental y aprendizaje autónomo.

Según el RRA (2019):

El aprendizaje en contacto con el docente es el conjunto de actividades individuales o grupales desarrolladas con intervención directa del docente (de forma presencial o virtual, sincrónica o asincrónica) que comprende las clases, conferencias, seminarios, talleres, proyectos en aula (presencial o virtual), entre otras, fortaleciendo el desarrollo de las competencias profesionales ...(Art. 27, p.8) El aprendizaje autónomo es el conjunto de actividades de aprendizaje individuales o grupales desarrolladas de forma independiente por el estudiante sin contacto con el personal académico. Las actividades planificadas y/o guiadas por el docente se desarrollan en función de su capacidad de iniciativa y de 
planificación; de manejo crítico de fuentes y contenidos de información; planteamiento y resolución de problemas; la motivación y la curiosidad para conocer; la transferencia y contextualización de conocimientos; la reflexión crítica y autoevaluación del propio trabajo, entre las principales. Para su desarrollo, deberán planificarse y evaluarse actividades específicas, tales como: la lectura crítica de textos; la investigación documental; la escritura académica y/o científica; la elaboración de informes, portafolios, proyectos, planes, presentaciones, entre otras. (Art. 28, p.8)

El aprendizaje práctico experimental es el conjunto de actividades (individuales o grupales) de aplicación de contenidos conceptuales, procedimentales, técnicos, entre otros, a la resolución de problemas prácticos, comprobación, experimentación, contrastación, replicación y demás que defina la IES; de casos, fenómenos, métodos y otros, que pueden requerir uso de infraestructura (física o virtual), equipos, instrumentos, y demás material, que serán facilitados por las IES. (Art. 29, p.8)

La diferenciación de aprendizajes descrita permite pautar conocimientos, actividades y metodologías pertinentes a los resultados de aprendizaje de la carrera, además que articulen con los principios educativos de la institución que se orientan como ya se abordó en: saber-saber (cognoscitivo), saber-hacer (práctico) y saber-ser (autonomía) (ver figura 5). De esta manera se busca producir una interrelación entre los conocimientos y la información para llegar a un aprendizaje sustancial o significativo. Así, se pone la mirada en el estudiante como eje del proceso de enseñanza-aprendizaje a diferencia de la educación tradicional con foco en el docente.

\begin{tabular}{|c|c|c|c|}
\hline Saberes Genéricos & Saber Saber & Saber Hacer & Saber Ser \\
\hline $\begin{array}{l}\text { Manejar el lenguaje } \\
\text { oral y escrito para } \\
\text { comunicarse con } \\
\text { pertinencia e } \\
\text { idoneidad en los } \\
\text { diferentes contextos } \\
\text { académicos, } \\
\text { cientificos, laborales } \\
\text { y profesionales. }\end{array}$ & $\begin{array}{l}\text { Comprender histórica y } \\
\text { criticamente la disciplina } \\
\text { para reflexionar sobre el } \\
\text { impacto del diseño en el } \\
\text { contexto social, asi como } \\
\text { su contribución a la } \\
\text { cultura desarrollo de las } \\
\text { organizaciones o } \\
\text { entornos productivos. }\end{array}$ & $\begin{array}{l}\text { Diagnosticar las } \\
\text { necesidades } \\
\text { del comitente, de una } \\
\text { oportunidad o de un } \\
\text { desafio existente en el } \\
\text { ámbito del Disenio de } \\
\text { Productos empleando } \\
\text { técnicas de } \\
\text { imvestigación }\end{array}$ & $\begin{array}{l}\text { Demostrar } \\
\text { compromiso con las } \\
\text { necesidades sociales y } \\
\text { medioambientales } \\
\text { desde una mirada } \\
\text { humanista }\end{array}$ \\
\hline $\begin{array}{l}\text { Aplicar las TIC para } \\
\text { optimizar sus } \\
\text { comunicaciones, } \\
\text { aprendizajes y el } \\
\text { desarrollo del } \\
\text { conocimiento, }\end{array}$ & $\begin{array}{l}\text { Relacionar los } \\
\text { fundamentos } \\
\text { epistemológicos y } \\
\text { metodológicos del } \\
\text { proceso de Diseño en } \\
\text { función del desarrollo } \\
\text { efectivo de las soluciones } \\
\text { de Diserio }\end{array}$ & $\begin{array}{l}\text { Desarrollar propuestas } \\
\text { en el ámbito de Diseño } \\
\text { de Productos dirigidas } \\
\text { tanto a entomos } \\
\text { artesanales como } \\
\text { industriales, } \\
5\end{array}$ & $\begin{array}{l}\text { Trabajar en un entorno } \\
\text { global y comprender } \\
\text { la importancia de la } \\
\text { conservacióny } \\
\text { transmisión del } \\
\text { patrimonio cultural } \\
\text { generando propuestas } \\
\text { de valor }\end{array}$ \\
\hline
\end{tabular}




\begin{tabular}{|c|c|c|c|}
\hline $\begin{array}{l}\text { Utilizar el } \\
\text { pensamiento lógico y } \\
\text { divergente para } \\
\text { comprender a los } \\
\text { sujetos y proponer } \\
\text { alternativas de } \\
\text { intervención ante los } \\
\text { fenómenos } \\
\text { productivos. }\end{array}$ & $\begin{array}{l}\text { Identificar expectativas, } \\
\text { demandas y necesidades } \\
\text { de los públicos } \\
\text { destinatarios y del } \\
\text { comitente mediante la } \\
\text { comprensión de los } \\
\text { factores humanos físicos, } \\
\text { cognitivos, visuales, }\end{array}$ & $\begin{array}{l}\text { Comunicar el proyecto } \\
\text { de mediante su } \\
\text { representación y } \\
\text { presentación adecuada a } \\
\text { los distintos } \\
\text { imvolucrados en el } \\
\text { proyecto de Disetlo, a } \\
\text { partir de instrumentos } \\
\text { manuales v digitales }\end{array}$ & $\begin{array}{l}\text { Identificar y } \\
\text { comprender y } \\
\text { necesidades del ser } \\
\text { humano mediante la } \\
\text { imvestigación y el } \\
\text { conocimiento } \\
\text { individual y social. }\end{array}$ \\
\hline $\begin{array}{l}\text { Trabajar de forma } \\
\text { colaborativa y en } \\
\text { redes con miras a la } \\
\text { creación de } \\
\text { ambientes de } \\
\text { aprendizaje abiertos, } \\
\text { interculturales que } \\
\text { estimulen la } \\
\text { creatividad y la } \\
\text { innovación. }\end{array}$ & $\begin{array}{l}\text { Identificar las tipologias, } \\
\text { caracteristicas, ventajas y } \\
\text { desventajas de los } \\
\text { materiales para establecer } \\
\text { procesos optimizados en } \\
\text { el disello de productos, } \\
\text { viabilizando costos y } \\
\text { determinando la menor } \\
\text { huella ecológica posible. }\end{array}$ & $\begin{array}{l}\text { Identificar y analizar las } \\
\text { causas del problema } \\
\text { previo a su solución, } \\
\text { utilizando herramientas } \\
\text { especializadas para el } \\
\text { establecimiento de } \\
\text { criterios de evaluación, } \\
\text { comprobacióny } \\
\text { aprobación. }\end{array}$ & $\begin{array}{l}\text { Gestionar el Diseffo } \\
\text { con ética como eje } \\
\text { transversal de todas } \\
\text { sus acciones. }\end{array}$ \\
\hline $\begin{array}{l}\text { Asumir la formación } \\
\text { a lo largo de la vida } \\
\text { como base para el } \\
\text { alcance de sus metas } \\
\text { reflexionando sobre } \\
\text { sus experienciasy } \\
\text { buscando la } \\
\text { construcción } \\
\text { permanente del saber. }\end{array}$ & $\begin{array}{l}\text { Comprender la } \\
\text { importancia y aplicación } \\
\text { del método proyectual en } \\
\text { Diseño. }\end{array}$ & $\begin{array}{l}\text { Investigar en diseño } \\
\text { con una propuesta } \\
\text { metodológica y teórica } \\
\text { pertinente, } y \\
\text { evidenciando el aporte } \\
\text { al campo disciplinar } \\
\text { para fortalecer la } \\
\text { actividad académica. }\end{array}$ & $\begin{array}{l}\text { Desarrollar la } \\
\text { capacidad de ser } \\
\text { dinámico, autónomo, } \\
\text { innovador para llevar } \\
\text { a cabo de manera } \\
\text { exitosa y eficiente la } \\
\text { práctica profesional y } \\
\text { el emprendedurismo. }\end{array}$ \\
\hline
\end{tabular}

Figura 5. Resultados de Aprendizaje del Plan de Estudios Q177 (Versión simplificada). Carrera de Diseño de Productos, Quito, PUCE. Fuente: Elaboración propia.

En tercer lugar se encuentra relevancia en la elaboración y reconocimiento del perfil académico-profesional de los docentes que constituyen la planta académica de la carrera, lo cual permite establecer asignaciones disciplinares a favor de la pertinencia y en busca de la calidad.

Vinculado a este punto interesa la gestión para la formación continua de los docentes que incluye procesos de capacitación, actualización y certificación de competencias laborales [y académicas] específicas, las que se ejecutan en forma de cursos, seminarios, talleres u otras actividades.

En el caso de la carrera de Diseño de Productos se ha formalizado desde la segunda mitad del año 2018 la asignación de un docente responsable de los procesos de capacitación, lo que ha permitido fortalecer las competencias generales y específicas del diseñador académico mediante líneas de formación (ver figura 6). 


\begin{tabular}{ll} 
2018 (segundo semestre académico) & Horas \\
\hline Curso de especialización en Teoria del Diseño & 20 \\
\hline Taller de Micotectura & 16 \\
\hline $\mathbf{2 0 1 9}$ (primer y segundo semestre académico) & Horas \\
\hline $\begin{array}{l}\text { Autodesk Fusion 360: Diseño, ingenieria y } \\
\text { fabricación digital básica de esquemas y prototipos }\end{array}$ & 40 \\
\hline Taller de gestión de clase a través de Moodle & 6 \\
\hline $\begin{array}{l}\text { Taller acabados para el diseño de productos } \\
\text { en madera con Sherwin Williams }\end{array}$ & 2 \\
\hline $\begin{array}{l}\text { Objetos para la vida cotidiana: Diseño territorio } \\
\text { e identidad }\end{array}$ & 20 \\
\hline Taller de Diseño y Construcción de zapatos & 40 \\
\hline $\begin{array}{l}\text { De la educación popular al Diseño participativo } \\
\text { Modelado 3D Básico y avanzado }\end{array}$ & 40 \\
\hline $\begin{array}{l}\text { Herramientas de investigación cualitativa para } \\
\text { proyectos creativos }\end{array}$ & 80 \\
\hline Neurodiseño de experiencias & 20 \\
\hline
\end{tabular}

Figura 6. Manual de Gestión de carrera de Diseño: Formación Continua (2019). Fuente: Elaboración propia.

Finalmente y dado el alcance del presente artículo se abordan en cuarto lugar las Tecnologías de la Información y Comunicación (TIC) que constituyen un factor de innovación de notable amplitud y que exigen un compromiso institucional, de la carrera y de su cuerpo docente que se ve favorecido por el uso de la Internet en pro de los requerimientos que la época, la sociedad y sus actores demandan.

En este sentido y a nivel universitario destacan de la PUCE los retos asumidos que suponen la organización de un proceso de enseñanza-aprendizaje mediante la incorporación y uso de herramientas actuales, digitales e innovadoras. Una de estas herramientas es la generación de ambientes o plataformas virtuales de aprendizajes en tanto espacios para la educación en la modalidad presencial, no presencial o mixta que permiten a los estudiantes disponer de condiciones favorables para su instrucción mediante el acceso a los recursos informativos y experiencias interactivas.

Según el mexicano Miguel Ángel Herrera (2006), pueden distinguirse en estos ambientes de aprendizaje elementos fundamentales como: “a) Un proceso de interacción o comunicación entre sujetos. b) Un grupo de herramientas o medios de interacción. c) Una serie de acciones reguladas relativas a ciertos contenidos. d) Un entorno o espacio en donde se llevan a cabo dichas actividades (p. 2)

Entre las ventajas ofrecidas por los ambientes virtuales como por ejemplo el implementado en la PUCE ${ }^{11}$, se encuentran variables como la flexibilidad, la rapidez y la sistematización. La flexibilidad ya que el estudiante puede establecer su propio ritmo y secuencia 
de aprendizaje, repetir lecturas, pausar videos para el aprendizaje autónomo, entre otros. Asimismo se propicia el trabajo colaborativo en equipos y se administra por el docente la atención y la estimulación sensorial para el desarrollo y logro de competencias mediante el uso de las TIC.

En el caso de la carrera de Diseño de Productos se define de manera obligatoria en cada semestre y para cada una de las 40 asignaturas que componen el Plan de estudios, la carga en el aula virtual de los temas, subtemas, horas por actividades de aprendizaje, instrumentos, fechas de evaluación y metodologías. Esto marca un orden desde el enfoque docente que se somete a seguimiento por parte de los estudiantes, quienes se empoderan de su programa de enseñanza a partir del control y la exigencia.

Este proceso de publicación de contenidos que se establece en línea dos veces por año, no solo es evaluado constantemente por los pares académicos internos de la carrera y las autoridades institucionales, sino que también fomenta la sistematización de prácticas, resultados y contenidos que hacen cada vez más eficiente la formación en base a procesos estables y asignaciones regulares de docentes a materias afines según su perfil.

El sistema de evaluación con recursos digitales es también una fortaleza del entorno virtual ya que posibilita entre otros elementos: a) mediante criterios de verificación valorar de manera homogénea e inmediata la entrega de resultados por trabajo de manera operativa y para trabajar con los estudiantes en la corrección y fortalecimiento de su desempeño estudiantil, b) mediante la entrega de evidencias virtuales minimizar el uso de material impreso y c) articular con dispositivos móviles: celulares, tabletas, computadoras lo que favorece la revisión periódica y el seguimiento evaluativo por el estudiantado desde cualquier ubicación, interna o externa a la institución.

Un rol importante en la transformación tecnológica lo tienen también las bibliotecas las cuales si bien aún mantienen su configuración tradicional, han evolucionado hacia modalidades digitales en paralelo que permiten a los estudiantes el acceso a los recursos educativos en línea. Un ejemplo de este modelo es el caso de la PUCE donde se fomenta efectivamente el uso de bibliografías electrónicas ${ }^{12}$ y textos digitalizados en línea que de manera preferencial conforman el microcurrículo o sílabo ${ }^{13}$.

Esto favorece la lectura simultánea de textos de interés por diversos alumnos, los cuales de disponerse únicamente en físico (impresos) no abastecerían la demanda académica con un solo ejemplar.

También se presenta como factor de cambio la posibilidad de reunir una amplia gama de materiales: libros, revistas, artículos en un solo lugar al cual se puede acceder de manera presencial o remota. En cualquiera de los casos se eliminan los riesgos de trabajar con informaciones y datos no verídicos al definirse una plataforma que filtra y selecciona para el estudiantado material relevante, actualizado y en base a su interés.

Para cerrar es importante reconocer que los procesos de innovación educativa son inconclusos ya que la demanda formativa involucra acciones de cambio y espacios de enseñanza-aprendizaje en constante modernización. 


\section{Conclusión}

Todo proceso de innovación educativa constituye un conjunto de decisiones y acciones que jerarquizan unos aspectos con respecto a otros de acuerdo a diferentes enfoques y prioridades. En el caso de la PUCE y particularmente de la carrera de Diseño de Productos la apuesta por el cambio no responde a una sola circunstancia, sino a varias que se entretejen social, política y económicamente.

Algunos de estos cambios en el camino de la innovación se concretan internamente en: a) el rediseño de la carrera enfocado en menos tiempo, generalmente 1 año menos que las carreras universitarias pares existentes históricamente lo que implica una selección de los contenidos sustanciales y significativos, b) la especificación y particularización de saberes enfocados en el estudiante: genéricos, vinculados al conocimiento, al desarrollo de acciones y al fomento de sus valores, c) el ajuste en asignaciones de materias según el perfil académico del docente a favor de la búsqueda por la pertinencia y la calidad en base a la experiencia profesional y a los años en la academia, d) la apropiación y uso eficiente de las Tecnologías de la Información y la Comunicación que enlazan a modo de red: infraestructura, programas asistidos por ordenador, bibliotecas, plataformas y clases virtuales, sistemas de evaluación, entre otras.

Como se puede ver las posibilidades para la innovación son amplias, sin embargo es importante monitorear el desarrollo y el avance de las acciones en base al cumplimiento de los objetivos planteados y para un período específico, también poniendo atención a las demandas de diversos actores en un contexto dinámico y con condiciones propias que constantemente implican el ajuste de estrategias. Los factores de innovación son oportunidades para el mejoramiento, lograr visualizarlos y poder manejar de modo eficiente sus cambios, alcances y articulaciones, nos permitirá llegar al objetivo de formar al mejor profesional de Diseño con herramientas apropiadas que amplíen sus posibilidades para ejercer laboralmente a nivel internacional y en el país.

En todo este proceso la responsabilidad y compromiso del docente supera el acto de sumarse al cambio de manera pasiva según leyes y normativas definidas. Saber ubicarse en el rol académico que se cumple: como puente con la empresa externa, como académico potenciador de capacidades y conocimientos, como emprendedor y comunicador de experiencias o como investigador líder de las prácticas educativas y transformador de modelos para el desarrollo académico y profesional, posibilita vencer la resistencia provocada por el temor a moverse del lugar ocupado o ganado.

La innovación proclama la necesidad de consolidar equipos docentes integrados que reconozcan el valor y pongan sobre si la responsabilidad del cambio, a la vez miren hacia afuera, en dirección a las tendencias educativas, los campos laborales, las rutas de emprendimiento para poder adelantarse y ajustarse a lo que acontece.

Para cerrar es importante puntualizar que si bien para lograr innovación la planeación y ejecución del proceso educativo es relevante, una adecuada selección de las herramientas para la enseñanza-aprendizaje de la mano de un proceso de evaluación constante pero sobre todo consciente posibilita garantizar y validar los beneficios esperados. 


\section{Notas}

1. Docentes, estudiantes, instituciones educativas, Estado, empresa público-privada, además de evaluadores o auditores internacionales, empresas que desarrollan y comercializan mundialmente contenidos educativos en línea, desarrolladores de programas computacionales y de servicios de evaluación y plagio en internet, entre otros.

2. https://www.puce.edu.ec/portal/carreras/diseno-de-productos/

3. El Sistema Nacional de Competencias (NVQ) establece niveles que permiten evaluar y relacionar el perfil de competencias de un puesto laboral con la capacidad del hombre para resolver las tareas asignadas, desempeñar acciones vinculadas a la gestión y a la solución de situaciones imprevistas y desenvolverse de modo eficiente en su entorno sociolaboral, es decir que se relacionan los atributos de las personas con su proceso de desempeño laboral y para el logro de resultados en base a objetivos.

4. Según la página web oficial y en línea del CES "El Consejo de Educación Superior... tiene como misión la planificación, regulación y coordinación interna del Sistema de Educación Superior del Ecuador, y la relación entre sus distintos actores con la Función Ejecutiva y la sociedad ecuatoriana" (CES, 12 de julio de 2012). Fuente: http://www.ces.gob.ec/ index.php?option=com_content\&view=article\&id=1\&Itemid $=140$

5. En el Mandato Constituyente No. 14, expedido por la Asamblea Nacional el 4 de noviembre de 2009, se establece un informe con el detalle de las categorías y parámetros de evaluación establecidos por Consejo Nacional de Evaluación y Acreditación (CONEA) del Ecuador. Para mayor información al respecto remitirse a https://www.educacionsuperior. gob.ec/wp-content/uploads/downloads/2012/07/Extracto_informe_CONEA.pdf

6. Una beca se define como la subvención total o parcial otorgada a personas naturales para que realicen estudios de educación superior. Las universidades ecuatorianas de manera particular pautan niveles de pagos diferenciados a partir de ofertas de beca que se concretan a modo general en: beca socio-económica, deportiva o cultural, de discapacidad o inclusión y de excelencia académica.

7. Para mayor información sobre los aranceles remitirse a: https://www.puce.edu.ec/documentos/2019/aranceles-puce-quito-2019-01.pdf

8. Se refiere a la publicación oficial que establece cambios posibles en los programas o carreras del Ecuador de manera especial por única vez.

9. Las IES pueden presentar estos rediseños de carreras o programas hasta el 21 de marzo del 2020.

10. Para ver la Guía en detalle remitirse a: http://www.ces.gob.ec/documentos/RPC-SO21-No_368-2019.pdf

11. https://eva.puce.edu.ec/

12. https://www.puce.edu.ec/biblioteca/

13. El sílabo se constituye en la herramienta de planificación y organización que contiene toda la información necesaria sobre la asignatura: su objetivo principal, unidades y contenidos, metodologías, instrumentos y técnicas de evaluación y referencias bibliográficas. Como se ha mencionado anteriormente la configuración microcurricular se estructura en base a las pautas del RRA del 2019. 


\section{Bibliografía}

Arnaz, J. A. (1981). La planeación curricular. México: Trillas.

Cañal de León, P. et al (2002). La innovación educativa. Pedro Cañal de León (coord.). Madrid: AKAL.

Carlos, G. J. (1998). Modelos e implicaciones curriculares de la educación basada en competencias (EBC). México: Facultad de Psicología, UNAM. Recuperado de https://www. academia.edu/7058922/Modelos_curriculares_de_la_Educaci\%C3\%B3n_Basada_en_ Competencias_EBC_

Castellanos, C. (21 de noviembre de 2019). Realidad y perspectivas de la Innovación Tecnológica en la Educación Superior [ponencia en línea]. En II Seminario Nacional "Los desafíos de la universidad en un mundo de cambios acelerados". Guayaquil, ESPOL: Observatorio Ecuatoriano de Buenas Prácticas de Dirección Universitaria Telescopi-Ecuador. Recuperado de http://telescopi.espol.edu.ec/index.php/seminario-2019/

CINDA (2011). Educación superior en Iberoamérica. Informe 2011. Santiago de Chile: RIL. Recuperado de http://admision.senescyt.gob.ec/media/2019/07/Oferta-2do-Semestre-2019_Digital_.pdf

Consejo de Educación Superior. (12 de julio de 2012). Misión, visión y objetivos (en línea). Ecuador: CES. Recuperado de http://www.ces.gob.ec/index.php?option=com_content \&view $=$ article\&id $=1$ \&Itemid $=140$

CONEA (4 de noviembre de 2009). Evaluación de desempeño institucional de las universidades y escuelas politécnicas del ecuador. Mandato Constituyente No. 14, Quito: Consejo Nacional de Evaluación y Acreditación (CONEA). Recuperado de https:// www.educacionsuperior.gob.ec/wp-content/uploads/downloads/2012/07/Extracto_informe_CONEA.pdf

Díaz, F. (1990). La evaluación cualitativa. México: Paidós.

Echavarría, L. (2010). Poder soberano y poder disciplinario. En Horizontes educativos: identidades políticas y educativas (Fuentes, S. y Piedad, O. Coord.), 141-160. México: UPN. Recuperado de http://xplora.ajusco.upn.mx:8080/xplora-pdf/Fuentes\%20Amaya,\%20Silvia.pdf .

Echavarría, L. y De los Reyes C. (2017). El modelo de educación basada en competencias: genealogía, análisis y propuestas. Congreso Nacional de Investigación Educativa COMIE. México, San Luis de Potosí. Recuperado de http://www.comie.org.mx/congreso/memoriaelectronica/v14/doc/1093.pdf

El Comercio. (9 de julio de 2019). El Cases explica proceso de acreditación de universidades en talleres [edición en línea]. Recuperado de https://www.elcomercio.com/actualidad/ proceso-acreditacion-universidades-escuelas-politecnicas.html.

Ecuador Universitario. (5 de septiembre de 2019). Desde el 2020 Ecuador eliminará la categorización de sus universidades. Recuperado de https://ecuadoruniversitario.com/ noticias-universitarias/desde-el-2020-ecuador-eliminara-la-categorizacion-de-susuniversidades/ 
El Universo. (16 de junio de 2019). La educación superior pública puede cubrir solo el 51\% de la demanda en Ecuador [edición en línea]. Recuperada de https://www.eluniverso. com/noticias/2019/06/16/nota/7377747/educacion-superior-publica-puede-cubrir-solo51-demanda-ecuador

Fägerlind, I., y Saha, L. J. (2016). Education and national development: A comparative perspective. Estados Unidos: Elsevier.

Consejo de Educación Superior (CES). (24 de junio de 2019). Guía Metodológica para la Presentación de Carreras y Programas. Quito. Ecuador: CES. Recuperada de http://www. ces.gob.ec/documentos/RPC-SO-21-No_368-2019.pdf

González, O. (1994). Curriculum: diseño, práctica y evaluación. La Habana, Cuba: Universidad de La Habana, CEPES.

Herrera, M., Jaramillo, J. y Mallarino, C. (2000). Licenciatura en educación física. Nuevo currículo para el 2001. Bogotá: Universidad Pedagógica Nacional (UPN).

Herrera, M. A. (2006). Consideraciones para el diseño didáctico de ambientes virtuales de aprendizaje: una propuesta basada en las funciones cognitivas del aprendizaje. Iberoamericana de Educación, (38) 5, 1-20. Recuperada de https://rieoei.org/historico/ deloslectores/1326Herrera.pdf

INEC (15 de julio de 2019). Boletín técnico No 03-2019, ENEMDU. Quito, Ecuador: INEC. Recuperado de: https://www.ecuadorencifras.gob.ec/documentos/web-inec/ EMPLEO/2019/Junio/Boletin_tecnico_de_empleo_jun19.pdf

Larrea de Granados, E. (abril, 2015). El currículo de la Educación Superior desde la complejidad sistémica. En Nuevos Horizontes de gestión en el sistema de Educación Superior ecuatoriano. Taller de difusión llevado a cabo por el Consejo de Educación Superior. Quito. Recuperado de http://www.ces.gob.ec/doc/Taller-difusion/SubidoAbril-2015/curriculo_es-sistemico $\% 20$-\%20e\%20larrea.pdf

La importancia del aprendizaje basado en competencias. (30 de noviembre de 2018). Universia. Recuperado de https://noticias.universia.net.mx/educacion/noticia/2017/01/26/1148881/ importancia-aprendizaje-basado-competencias.html

Margalef, L. y Arenas, A. (2006). ¿Qué entendemos por innovación educativa? A propósito del desarrollo curricular. Perspectiva educacional, N ${ }^{\circ} 47,15-31$. Recuperada de https:// www.redalyc.org/pdf/3333/333328828002.pdf

Metro Ecuador. (27 de febrero de 2019). Senescyt: Institutos superiores técnicos y tecnológicos otorgarán títulos de tercer nivel. Por Andrea Martínez (ed.). Recuperado de https://www. metroecuador.com.ec/ec/estilo-vida/2019/02/27/senescyt-titulos-tecnicos-tecnologicostercer-nivel.html

Modelo Educativo de la PUCE. (6 de marzo de 2017) Quito, Ecuador: PUCE. Recuperado de https://www.puce.edu.ec/intranet/documentos/Reglamentos/PUCE-ModeloEducativo-06-03-2017.pdf

Observatorio Ecuatoriano de Buenas Prácticas de Dirección Universitaria. (21 de noviembre de 2019). Retos y desafíos de la innovación tecnológica en la Educación Superior. Ecuador: Telescopi \& ESPOL. Recuperado de http://www.espol.edu.ec/es/ noticias/retos-y-desaf\%C3\%ADos-de-la-innovaci\%C3\%B3n-tecnol\%C3\%B3gica-enla-educaci\% $\mathrm{C} 3 \% \mathrm{~B} 3 \mathrm{n}$-superior 
Pérez, M. del R., Orozco, C. y Cruz, L. (Mayo de 2013). Un análisis de los factores de innovación curricular. Pistas Educativas, 101. México: Instituto Tecnológico de Celaya. Recuperado de https://www.researchgate.net/publication/320271292_Un_analisis_de_ los_factores_de_innovacion_curricular

Reglamento General a la Ley Orgánica de Educación Superior, Registro oficial N ${ }^{\circ} 298$ (12 de octubre de 2010). Presidencia de la República del Ecuador. Recuperado de https:// www.educacionsuperior.gob.ec/wp-content/uploads/downloads/2014/03/LEY_ORGANICA_DE_EDUCACION_SUPERIOR_LOES.pdf

Reglamento General a la Ley Orgánica de Educación Superior, Registro oficial N 503 (6 de junio de 2019). Presidencia de la República del Ecuador. Recuperado de https:// www.caces.gob.ec/

Rosario, V. M. y Alvarado, M. (2019). Innovar en el Desarrollo Curricular: una propuesta metodológica para la Educación Superior. Bloomington: Palibrio.

Secretaría General de la PUCE. (28 de noviembre de 2018).Tabla de aranceles, matrículas $y$ derechos. Quito: PUCE. Recuperada de https://www.puce.edu.ec/documentos/2019/ aranceles-puce-quito-2019-01.pdf

UNESCO. (2016). Innovación educativa, texto 1. Lima: UNESCO. Recuperado de https:// unesdoc.unesco.org

Vargas, M. R. (2008). Diseño curricular por competencias. México: Asociación Nacional de Facultades y Escuelas de Ingeniería (ANFEI). Recuperado de https://www.gob.mx/cms/ uploads/attachment/file/182548/libro_diseno_curricular-por-competencias_anfei.pdf

Vidal, M. J. et al (2016). Educación basada en competencias. Educación Médica Superior, 30 (1), 1-8 Recuperado de http://scielo.sld.cu/scielo.php?script=sci_arttext\&pid=S086421412016000100018\&lng=es\&tlng=es.

\begin{abstract}
The following article describes some of the main reasons that drive curricular change in higher university education at the Pontifical Catholic University of Ecuador. This is mainly due to national socio-economic circumstances and educational reforms of a legislative nature. It is then investigated in the proposal of the curricular redesign that is established in the year 2019 for the Product Design career of the institution in question and with an approach that focuses on the strategies within it in the framework of innovation educational Factors such as virtual environments, Information Technology and Communication, the decrease in academic periods are hand in hand with a selection of substantial content, among others. Finally, it is concluded that the innovation factors developed seem to be organized by layers defined from national, institutional (PUCE) instances and to a lesser extent within the career itself, because of which one must reflect on the way in which the latter is due appropriate the discourse and change process to articulate their own innovative and significant actions.
\end{abstract}


Keywords: academic curriculum - Product Design - Higher Education - university training - curriculum innovation.

Resumo: $\mathrm{O}$ artigo a seguir descreve alguns dos principais motivos que impulsionam a mudança curricular no ensino superior universitário na Pontificia Universidad Católica del Ecuador. Isso ocorre principalmente à luz das circunstâncias socioeconômicas nacionais e das reformas educacionais legislativas. Em seguida, é investigado na proposta do redesenho curricular estabelecido em 2019 para a carreira de Design de Produto da instituição em questão e com uma abordagem focada nas estratégias dentro dela no âmbito da inovação. educacional.Fatores como ambientes virtuais, Tecnologias da Informação e Comunicação, a diminuição dos períodos acadêmicos, são tratados por uma seleção de conteúdos substanciais, entre outros. Por fim, conclui-se que os fatores de inovação desenvolvidos parecem ser organizados por camadas definidas a partir de instâncias institucionais nacionais (PUCE) e, em menor grau, dentro da própria carreira, motivo pelo qual é necessário refletir sobre a maneira pela qual esta última pode ser apropriarse do discurso e do processo de mudança para articular suas próprias ações inovadoras e significativas.

Palavras chave: currículo acadêmico - Design de Produto - Ensino Superior - formação universitária - inovação curricular.

[Las traducciones de los abstracts fueron supervisadas por el autor de cada artículo] 